\title{
Configurations of renewable power generation in cities using open source approaches: with Philadelphia case study
}

\author{
Joseph Ranalli ${ }^{\mathrm{a}}$, Alaa Alhamwi ${ }^{\mathrm{b}}$ \\ ${ }^{a}$ Penn State Hazleton, 76 University Drive, Hazleton, PA, USA 18202 \\ ${ }^{b}$ German Aerospace Center (DLR) Institute of Networked Energy Systems, Carl-von-Ossietzky-Straße 15, 26129 Oldenburg, Germany
}

\begin{abstract}
In this paper, an open source tool is introduced to represent urban energy infrastructure in the City of Philadelphia, and different renewable energy scenarios are compared with respect to minimization of the standard deviation of the residual load. Renewable energy sources play a critical role in the world's ongoing energy transition in response to climate change. Urban Energy Systems may be particularly sensitive to this transition due to the high energy demand density associated with urban environments. Open energy analysis and modeling tools can provide important information that can be used by urban energy planners, policy makers, and other stakeholders during this transition. In the present study, we apply FlexiGIS, an open energy modeling tool developed in a European context, to a case study in the City of Philadelphia. Due to the importance of open access to energy data, we pay particular attention to open energy data sources. Notably, OpenStreetMap was incomplete in its spatial coverage, but alternate open data resources were identified. This work conducts an optimization of the renewable energy mix to minimize the amount of balancing energy required for the residual load. We observe that Philadelphia has an optimal mix of renewables that favors a roughly even share of wind and solar, but that, compared to a previous case study in Oldenburg, Germany, requires more balancing energy at comparable levels of renewable penetration.
\end{abstract}

Keywords:

FlexiGIS platform, Urban Energy Systems (UES), Geographic Information Systems (GIS), OpenStreetMap (OSM), open source data and models, optimal mix.

\section{Introduction}

Transitioning from traditional energy generation toward carbon-neutral renewable energy sources will be necessary to mitigate future global impacts of climate change. This transition is expected to present novel challenges to planning and operations of existing electricity systems. Urban Energy Systems (UES) are areas of particular interest in this respect, because the concentration of loads in urban centers can serve to amplify stresses placed on a system as renewables are introduced. By the middle of this century, the world's urban population is expected to double that of 2008 (1). Cities account for $65 \%$ of global energy demand and $75 \%$ of carbon emissions, with an increasing trend (2). Although the role of cities in shaping the trajectory of climate change has been recognized in some international agreements like the New Urban Agenda (3), implementation strategies have not received enough recognition in global political agendas (4). The role of cities in leading the transition toward renewable energy is motivated by drivers that REN21 categorizes as environmental, socio-economic and energy security or governance (2).

Policies and planning related to energy transition in urban environments may often begin at the local level. In Germany, bottom-up insights and experiences are gaining increasing attention as ways to ensure a successful energy transition, "En- ergiewende," by engaging cities. Integration of local urban renewable energy resources has been treated as a vital milestone for the sustainable transformation in German cities (5). As an example, the city of Emden has set a target of covering $100 \%$ of its energy demands from renewable sources by 2030 (6). In addition, the city of Herten has been using a virtual energy storage system that includes available storage systems and energy generation, allowing a higher share of urban renewables (7). In the US, cities have also taken a role in forming energy policy. For example, the City of Philadelphia has adopted a plan to reduce carbon pollution by $80 \%$, and to transition to $100 \%$ carbon-free electricity by 2050 (8). Studies have demonstrated that energy policies are subject to politicization and resistance as they begin to threaten existing energy industry actors (9). However, modeling of energy transition in the US suggests that continued growth of the renewable share of electricity generation is expected to be driven by economic factors (10).

The optimal design of city-integrated renewable energy systems needs systematic modeling approaches and platforms like in (11). Hence, analyses of UES based on renewable energy possess a fundamentally spatial nature (12). While loads have always had a distributed character, renewable systems introduce a spatial character to generation and resource availability as well. This is particularly true for power generated by solar photovoltaics (PV) and wind turbines. Unlike traditional 
fuel resources, which can be collected and concentrated for utilization, renewable resources like solar and wind are available in relatively diffuse, hard to aggregate quantities, that are distributed throughout the environment. Consequently, generation facilities based on exploiting these resources must be distributed as well. This distributed generation may require the development of advanced control strategies to maintain power quality and grid stability (13). Despite presenting operational challenges, this situation also represents a significant opportunity; future energy systems that are designed with distributed load, generation and storage in mind may be able to benefit from spatial matching of these elements and offer positive benefits to system operation. Energy models and tools integrated in a Geographic Information System (GIS) platform offer many advantages in this regard. GIS has been considered as a useful tool that facilitates connecting data with geography. It allows multilayer and realistic representation of urban energy systems, as well as links and interdependencies between different layers. Research is still needed to develop a better understanding of the interplay between the various elements of UES with renewables and how their performance can best be modeled and anticipated. Using GIS techniques, this research combines both temporal and spatial parameters of urban geometries, linking them to real world applications utilizing solely open source urban energy datasets. Ultimately, our ability to efficiently plan and manage the increasing penetration of renewable generation facilities into UES relies on the availability of data and tools to support reliable, high confidence technical analysis and monitoring of these systems, including the crucial spatial dimension.

While data and tools to support renewable energy analysis must be spatial in nature, it is also desirable for these resources to have an open character (14). Considering these endeavors from a perspective that considers all goals of sustainable development (15), open access is able to reduce barriers for policymakers, planners and other stakeholders and can provide an equitable platform to investigate energy scenarios, so that decision making can be driven by sound scientific knowledge. Modern, city-level renewable energy policies are made by entities of all sizes, and in all areas of the world (2). Consequently, decision makers exhibit a great diversity in terms of financing and resources available to support these types of planning activities. While no one tool may be available to enable out-of-the-box analysis of all types of energy scenarios, operating within an open framework (16) allows tools to be easily adapted for local contexts utilizing available data. Opening up UES tools will enable researchers, students and academics to further develop and create new urban energy models saving time and resources.

This study makes an original contribution to the field by testing application of an existing open UES modeling tool in a new context that is subject to different data constraints than those for which it has been previously demonstrated. FlexiGIS (17) is an open access spatial tool that was developed for modeling of UES. This tool, however, was developed in a European context, relying on commonly available open data sources therein. In this study, we demonstrate for the first time the applicability of FlexiGIS in a case study in the US with different data avail- ability constraints. In conducting this case study, we identify alternate data sources that support application of this model in the US context demonstrated here. One of FlexiGIS's key computational capabilities is the optimization of renewable energy mix in an urban context. Though this modeling approach has already been reported in the literature, we have improved upon the existing formulation by deriving an analytical form of the optimization that can now be used to provide direct insight into the optimum renewable energy mixture's dependence on its input data. For the first time, we are also able to provide an open source implementation of this calculation in the supplementary materials, to facilitate its application.

The remainder of this paper is structured as follows; an overview of selected UES tools and the FlexiGIS model are introduced in Section 2. Section 3 discusses input data collection for UES modeling. The developed methodology and data processing for the case study are presented in Section 4. Section 5 presents the resultant data and provides an analysis and discussion of its implications. Section 6 contains the conclusions and recommendations. Finally, supplementary materials and the developed scripts are provided in the Appendix.

\section{Existing Tools and Platforms}

This section provides an overview of some selected urban energy models that have been developed to investigate different aspects of energy systems in cities. Most of the developed tools are designed for a particular urban energy sector or application. The Open Source Energy Modeling System (OSeMOSYS) framework was created for modeling developing countries at the national level (18). An extended and customized version of OSeMOSYS was introduced by (19) for the continental US scale. It aims to investigate US electricity infrastructure pathways by 2050. Another study (20) introduced a mathematical model to explore the photovoltaic potential as an energy supplier for charging electric vehicles in cities. The authors in (21) present a Bayesian hierarchical model to estimate the PV potential in residential areas.

CitySim is a tool developed by Robinson et al. (22) for the optimization of building-related resource flows for sustainable urban planning. Nitkiewicz et al. (23) describe a data-driven urban energy simulation tool (DUE-S), introduced primarily to predict the energy consumption of multi-scale urban energy modeling workflows in US settings. Another model, Synthetic City (SynCity), facilitates the integrated modeling of urban energy systems (24).

SimStadt is a model that provides a platform for three dimensional city models and visualization support (25). CityGML is a standard data model developed to store digital 3D models of cities. It allows a multi-scale representation of spatial data infrastructure in cities (26). SUNtool (Sustainable Urban Neighborhood modeling tool) supports urban planners in optimizing the sustainability of urban neighborhoods (27). HUES stands for Holistic Urban Energy Simulation and allows investigation of the relationship between district size and the sizing of the 
necessary infrastructure (28). An open source model, called urbs, can be used to investigate energy scenarios and capacity expansion in urban energy systems (29). Finally, uesgraphs (30) introduces methods to graphically represent and analyze energy network structures and handle their datasets in a Python graph structure.

Based on a comprehensive review of the state of the art of GIS based models for urban energy systems, Alhamwi et al. (17) demonstrated that growing research interest exists in modeling urban energy systems using Geographic Information Systems (GIS). The authors in (17) identified a gap in the literature related to the integration of flexibilization assessment using GIS techniques at the city scale, and a platform called FlexiGIS was developed to fill in this gap.

\subsection{FlexiGIS Overview}

FlexiGIS stands for Flexibilization in Geographic Information Systems (GIS) ${ }^{1}$. FlexiGIS is an open source GIS-based platform for the optimization of flexibility options in urban areas, and has previously been applied and showcased in the literature in a primarily European context (31). The FlexiGIS platform consists of three main modules:

- The first module establishes the underlying spatial urban energy infrastructure like streets, buildings, and available urban renewable power capacities. It downloads raw geo-datasets from OpenStreetMap for a specific urban area under investigation. Subsequent steps include geoproceessing, extracting, filtering and clustering of the georeferenced urban energy infrastructure data (17).

- The second module adds the temporal dimension to the first. It develops simplified models to simulate local electricity consumption and on-site renewable power generation (32).

- The third module uses the datasets generated in the first two modules and other techno-economic datasets to perform a cost optimization of flexibilization technologies, like storage, and identifies the potential areas for optimized decentralized storage in urban settings (33).

The resultant datasets from all three modules can be visualized using any GIS client, including open tools such as QGIS ${ }^{2}$. Furthermore, FlexiGIS systematically investigates different scenarios of self-consumption and analyzes the characteristics and roles of flexibilization technologies in promoting higher energy independence levels in cities (31). The FlexiGIS platform aims to link the simulations of urban energy infrastructure to real world applications. It is designed to be modular and current development is underway to automate all its modules so that it can be easily extended to other areas.

\footnotetext{
${ }^{1}$ https://github.com/FlexiGIS/FlexiGIS.git

${ }^{2}$ https://www.qgis.org/
}

Though FlexiGIS has been described previously in the literature, it has not demonstrated in the US. Here, we apply FlexiGIS to a case study in the US (namely, the City of Philadelphia, Pennsylvania), in order to test the extensibility of the platform. In particular, it was found that the City of Philadelphia does not have a complete dataset available in OpenStreetMap, the open geodata source on which FlexiGIS relies. Thus, investigation of alternate open data sources is necessary to evaluate FlexiGIS in this context. In addition to extension of the tool, we also use this case study as an opportunity to draw a comparison between the present results and prior data from Oldenburg, Germany (32).

\section{Data Availability}

The City of Philadelphia, located in the southeast corner of the state of Pennsylvania, is the most populous city in the state and the sixth largest city in the US, based on 2019 data (34). In terms of energy services, the regional transmission organization (RTO) responsible for most of the state of Pennsylvania as well as several neighboring states is PJM. The PJM region is broken into a number of load areas based on local distribution utility companies. Among these is PECO (Figure 4), the local utility serving Philadelphia and its surrounding area. The area served by PECO had a 2010 Census population of around 3.8 million, around $40 \%$ of which (1.5 million) lived within the City of Philadelphia (35).

Philadelphia's status as a large metropolitan area, and the availability of open data related to the city and its energy systems make it a convenient case study for urban energy system analysis and FlexiGIS. In the following section, we will summarize some open data sources related to UES for the City of Philadelphia (and surrounding PECO region) that could be used as inputs for energy modeling. We targeted the year 2017, representing the most recent year for which all types of time series data were presently available.

\subsection{Defining Openness}

Any discussion of open data requires specificity in the definition of open. Relative to the context of user access to services, openness may be described from a dual perspective of cost and user rights. Some data sources and tools in this framework may be described as universally open: they can be accessed without cost and are subject to a permissive license that includes the right to redistribute the data (e.g. the Open Database License ${ }^{3}$ used by OpenStreetMap). Other data sources may be free of cost, but are still subject to a license that affects users rights to reuse or redistribute the data. As stated previously, in the context of tools for UES modeling and analysis, we consider openness to primarily impact the ability of users to access and utilize tools to gather information and apply the analyses to their own unique context. As such, it is desirable that tools

\footnotetext{
${ }^{3}$ https://www.opendatacommons.org/licenses/odbl/
} 
(such as FlexiGIS) are both free of cost and have permissive licenses that allow users to access them for any purpose. However, we will deem input data sources that are publicly available without cost to qualify as sufficiently open for the purposes of this discussion, in that it is not inherently necessary for users to redistribute source data in order to reduce barriers to access of this knowledge. All the data sources described in the following sections meet this sufficient criterion of being accessible by the public free of charge, so that they can serve as inputs to a general set of analyses using FlexiGIS.

\subsection{Energy Infrastructure}

We gathered data on existing power generation facilities from the US Energy Information Agency (EIA) website ${ }^{4}$. This data includes all power generation facilities in the US with a nameplate capacity greater than $1 \mathrm{MW}$. Additionally, shape files were available from the Department of Homeland Security for transmission lines and electrical substations ${ }^{5}$ in the US. A visualization of all the energy infrastructure data for the PECO region is shown in Fig 1.

\subsection{Structures and Land Use}

In previous studies on FlexiGIS, OpenStreetMap (OSM) was used in load calculations as a source of detailed information about buildings and their intended use (17). When considering this data source for use in the City of Philadelphia, it was discovered that the OSM building data is somewhat incomplete, so other sources were considered. Generally, a great deal of open spatial data related to buildings and land use are available for the US, but data sources are somewhat fragmented, as most of this data is distributed at the state or city level. In this case, data for the State of Pennsylvania is available from the Pennsylvania Spatial Access Database (PASDA) ${ }^{6}$. Shape files of building footprints for the City of Philadelphia, and their associated land use tags, were acquired from PASDA.

To quantify the degree to which OSM data for the City of Philadelphia was available, a comparison was made between the building data from OSM (applying FlexiGIS) and that obtained from PASDA. Within the city boundaries, OSM was found to contain fewer than $10 \%$ of the buildings included in the PASDA dataset (45,126 buildings OSM to 539,558 in PASDA). When considering building footprint area, the ratio is slightly higher (22\%: $15.2 \mathrm{~km}^{2}$ area to $\left.67.6 \mathrm{~km}^{2}\right)$, indicating that OSM preferentially contains large buildings. This is evident in the comparison map images shown in Fig. 2 for OSM and Fig. 3 for PASDA. It is clear that OSM is missing large regions of buildings, especially in the upper half of the map. It may also be useful to compare the total area for which land use is specified between the two sources. In this case we find an area coverage of $107 \mathrm{~km}^{2}$ for OSM to $368 \mathrm{~km}^{2}$ for PASDA (OSM has $29 \%$

\footnotetext{
${ }^{4}$ https://www.eia.gov/maps/layer_info-m.php

${ }^{5}$ https://hifld-geoplatform.opendata.arcgis.com/datasets

${ }^{6} \mathrm{https}: / /$ www.pasda.psu.edu
}

coverage). Given that PASDA data is provided in the public domain, incorporating this data resource would improve the suitability of OSM as a tool for UES modeling, however at present, OSM could be said to substantially underrepresent the number of energy-using buildings in Philadelphia.

\subsection{Electrical Demand}

A model of the electrical demand is necessary to estimate the impact of renewable energy generation on the grid baseline. Previous contributions have utilized standard load profiles in order to estimate the hourly energy load (32). Standardized building load profiles are available for Typical Meteorological Year version 3 (TMY3) locations throughout the US from the Department of Energy Office of Energy Efficiency and Renewable Energy ${ }^{7}$. These load profiles are based on 16 types of reference commercial buildings, as well as residential buildings. In principle, these load profiles principle be used to simulate loads based on the building footprint area and land use type.

Alternatively, measured electricity demand data may be available from various RTOs (or ISOs) in the US. For example, PJM provides access to historical hourly metered load data by individual load area ${ }^{8}$. In this study, we used hourly load data in the PECO load area (which includes Philadelphia) to characterize electrical demand in the case study region (36). This data is subject to copyright by PJM, but is accessible by the general public at no cost via the Data Miner 2 tool.

\subsection{Resource Data}

Renewable resource time series data were used as inputs for renewable energy system (solar and wind) modeling. These data were obtained from the National Solar Radiation Database $(\mathrm{NSRDB})^{9}$. An hourly time step was chosen for the resource data, in order to match the time step of the load data. Time stamps in the NSRDB resource data represent instantaneous values, and are measured at the mid-hour point (e.g. 8:30 represents the hour from 8:00 to 9:00). NSRDB data provide time series for components of solar irradiance (GHI, DHI, DNI) as well as meteorological parameters such as ambient temperature, pressure, wind speed and wind direction. Solar resource data are based upon the Physical Solar Model (PSM) v3 (37), while additional meteorological parameters are based upon MERRA2 .

Wind speed data that can be used for wind power generation modeling purposes are available for the US in the Wind Integration National Dataset (WIND) (38). WIND data is available for the years 2007-2013. As mentioned, NSRDB solar irradiance files also include wind speed based on MERRA-2, but no detail is available about the altitude for which it is computed. Wind data can also be obtained directly from MERRA-2, which provides access to a several meteorological parameters at various

\footnotetext{
${ }^{7}$ https://openei.org/datasets

${ }^{8}$ http://dataminer2.pjm.com/feed/hrl_load_metered/definition

${ }^{9}$ https://nsrdb.nrel.gov/
} 


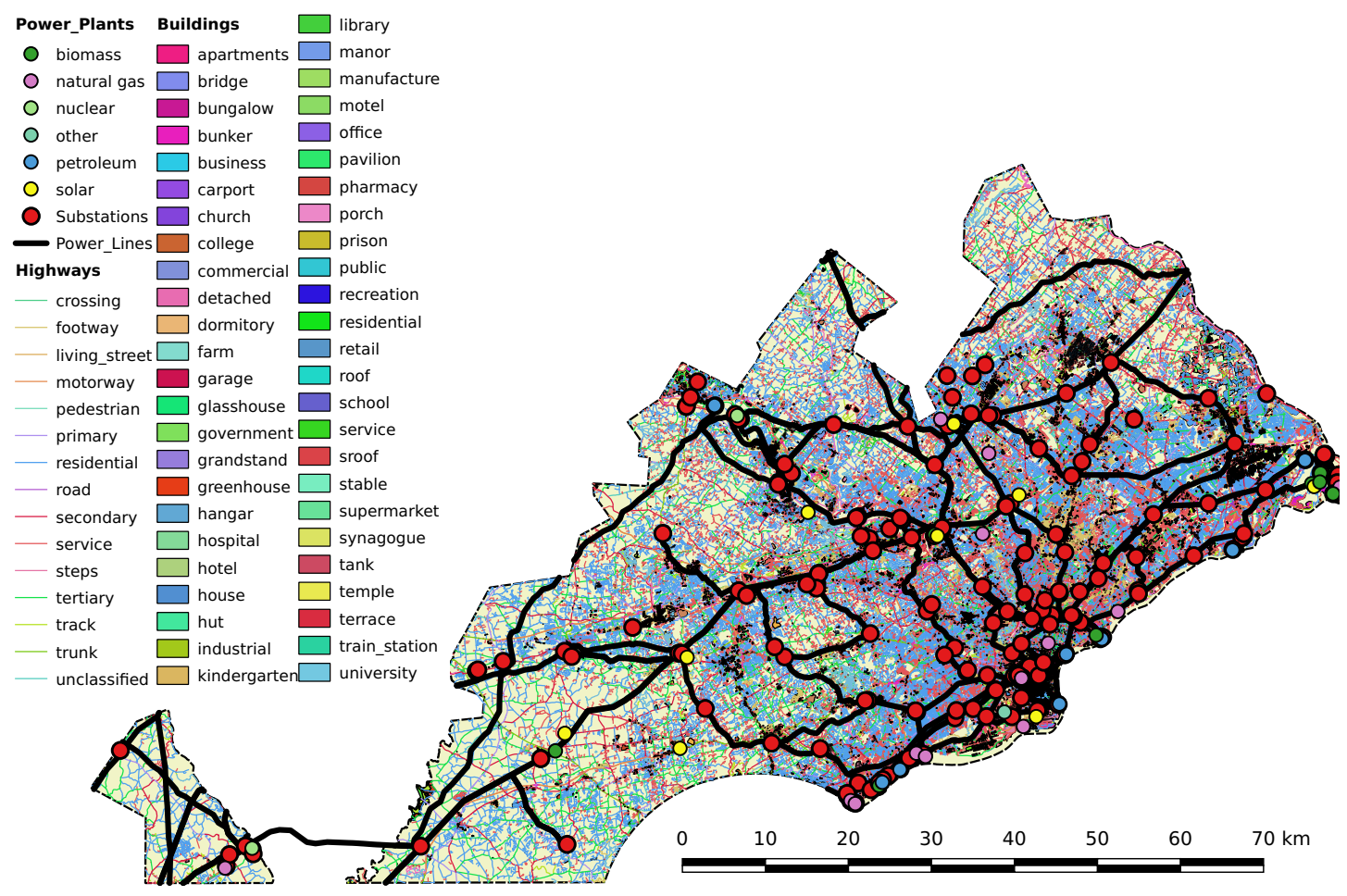

Figure 1: Energy infrastructure data sets for PECO service region extracted from OpenStreetMap using FlexiGIS. Power lines and substation obtained via EIA. Credits: OpenStreetMap contributors, QGIS Desktop.

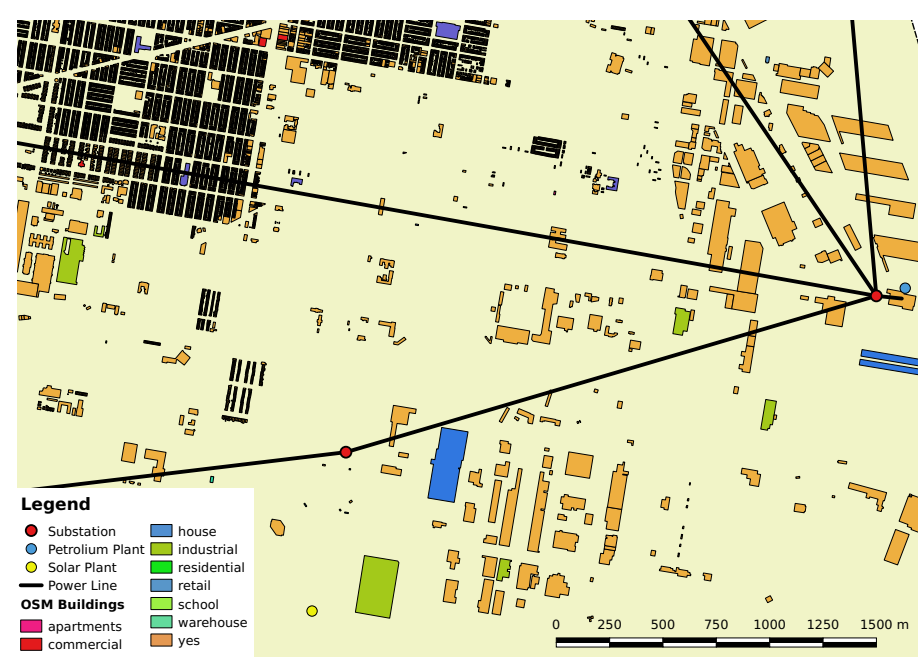

Figure 2: Zoom in - urban energy infrastructure datasets in Philadelphia. Buildings extracted from OSM using FlexiGIS. Credits: OpenStreetMap contributors, QGIS Desktop.

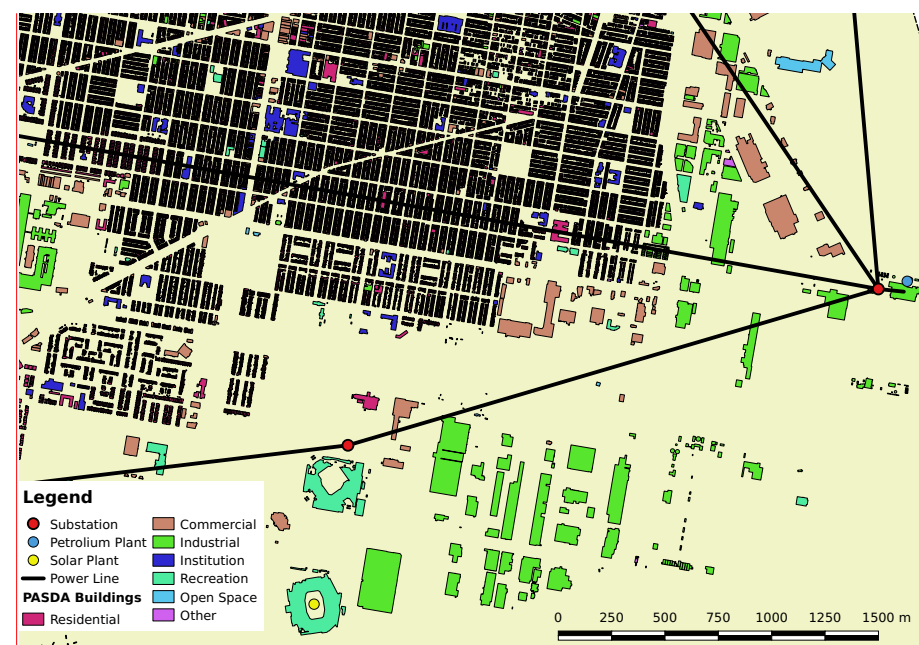

Figure 3: Zoomed in urban energy infrastructure datasets in Philadelphia. Buildings extracted from PASDA.

time scales (39). In the present study, we relied on hourly values of wind speed at an altitude of $10 \mathrm{~m}$, obtained from MERRA-2 via $\mathrm{SoDa}^{10}$.

${ }^{10} \mathrm{http}: / /$ www.soda-pro.com/web-services/meteo-data/merra 


\section{Case Study}

As stated previously, due to the incompleteness of OSM data in some cases, application of FlexiGIS in the US may require utilization of alternate data sources. As such, we have conducted a case study of its application for the optimization of renewable energy systems in the City of Philadelphia. Some previous contributions developed simplified models to investigate the optimal mix of renewable power generation at national level (40) and continental scales like in (41). The model was also utilized to assess continental scale storage needs with excess generation (42) and to consider the optimal mix of generation with regard to seasonal effects (43). The methodology used for this case study will be described in the following sections.

\subsection{Modeling Approach}

Previous work utilized FlexiGIS to compute the optimum mix of renewables from a meteorological perspective by analyzing the residual load with different balances of wind and solar, with any unmet energy demand purchased from the grid (32). In this formulation, the residual load, $R$, can be defined as the difference between the demand, $L$, and the renewable generation, $W$ and $S$, for wind and solar respectively:

$$
R(t)=L(t)-W(t)-S(t)
$$

We wish to cast this equation into a form that allows for variable levels of renewable generation relative to the annual load. We do this by normalizing to the mean annual load, $\langle L\rangle$, and introducing the mean annual wind, $\langle W\rangle$, and solar, $\langle S\rangle$, generation. We can then define explicit coefficients that represent the fraction of the annual load met by the renewables: $\alpha$ for wind fraction, and $\beta$ for solar fraction.

$$
R_{n}(t)=\frac{L(t)}{\langle L\rangle}-\frac{\langle W\rangle}{\langle L\rangle} \frac{W(t)}{\langle W\rangle}-\frac{\langle S\rangle}{\langle L\rangle} \frac{S(t)}{\langle S\rangle}=\frac{L(t)}{\langle L\rangle}-\alpha \frac{W(t)}{\langle W\rangle}-\beta \frac{S(t)}{\langle S\rangle}
$$

We can consider this equation for various mean levels of renewable generation ( $\alpha$ and $\beta$ ) relative to the annual load, such that the remainder would be imported from the grid. We can compute the fraction of the mean annual load that must be imported from the grid, or met by non-distributed renewables (e.g. Hydro/Biofuel power), as follows:

$$
\langle C\rangle=1-\alpha-\beta
$$

Previous work has considered the standard deviation of $R_{n}, \sigma_{R}$, to represent a cost function that serves as a surrogate for the cost of storage or other dispatchable generation necessary to accommodate fluctuations in the residual demand. A value of $\sigma_{R}=0$ would imply that wind and solar combined perfectly meet the demand at all times, while larger values indicate that though renewables might meet the mean annual demand, instantaneous overproduction must be shifted, e.g. by storage, to meet demand in times of undergeneration.

Previous studies have used a computational approach to determine the cost function at various renewable mix conditions.
However, the cost function can more conveniently be represented as the grand sum of all terms in the covariance matrix. Here, we use a generic $\sigma_{x}$ to represent the standard deviation in time series $x$, while $\sigma_{x y}$ indicates the covariance between two time series, $x$ and $y$. The subscripts $l, w$, and $s$ identify the load, wind and solar time series respectively. The sum is as follows:

$$
\sigma_{R}^{2}=\sigma_{l}^{2}+\alpha^{2} \sigma_{w}^{2}+\beta^{2} \sigma_{s}^{2}-2 \alpha \sigma_{l w}-2 \beta \sigma_{l s}+2 \alpha \beta \sigma_{w s}
$$

or, by substituting the definition of the correlation coefficient, $\rho:$

$\sigma_{R}^{2}=\sigma_{l}^{2}+\alpha^{2} \sigma_{w}^{2}+\beta^{2} \sigma_{s}^{2}-2 \alpha \rho_{l w} \sigma_{l} \sigma_{w}-2 \beta \rho_{l s} \sigma_{l} \sigma_{s}+2 \alpha \beta \rho_{w s} \sigma_{w} \sigma_{s}$

Inspecting Eq. 5, we see that the mathematical description agrees with an intuitive understanding of how renewable generation offsets the load; the cost function grows with increasing variance in the wind and solar generation, but can be reduced when the time series of wind and solar generation are correlated with the load. Additionally, we see that it is possible to estimate the effects of various fractions of wind and solar generation $(\alpha$ and $\beta$ ) on the cost function based solely on knowledge of the statistical properties of the three time series, $L(T), W(t)$, and $S(t)$, normalized to their means. Details of how these time series were computed will be provided in the subsequent sections.

\subsection{Data Processing}

The load timeseries was obtained directly from PJM via Data Miner 2, as discussed in section 3.4. The data consisted of hourly demand in MW for the year 2017. The load data were normalized to their annual mean, as in Eq 2. This data is an aggregate for the entire PECO region. For wind and solar resources, which exhibit inherent spatial variability, time series were aggregated to produce a single representative time series for generation in the entire region.

In the case of the PECO region, a total of 9 solar power facilities greater than $1 \mathrm{MW}$ in rated capacity were present (see section 3.2). Of these sites, 5 that spanned the entire region were selected to represent the spatial distribution of the solar resource (see Fig 4). No wind installations larger than $1 \mathrm{MW}$ were found within the PECO footprint, so the same 5 sites were also used as sources for wind speed data. Solar irradiance data were obtained from the NSRDB, as described in section 3. Wind speed data were obtained from MERRA-2 at a height of $10 \mathrm{~m}$ and were extrapolated to $100 \mathrm{~m}$ using the common Hellmann exponential relationship (44):

$$
\frac{v}{v_{0}}=\left(\frac{h}{h_{0}}\right)^{a}
$$

where $v$ is the velocity of interest at height $h, v_{0}$ is the known velocity at height $h_{0}(10 \mathrm{~m}$ in this case) and $a$ is the friction coefficient, which depends on the ground condition. For this study, a friction coefficient of 0.3 was used.

For both solar and wind generation, resource data from the 5 distributed sites were used as input to power generation models. The hourly power produced by each of the 5 sites was averaged to generate a single aggregate time series for each type 


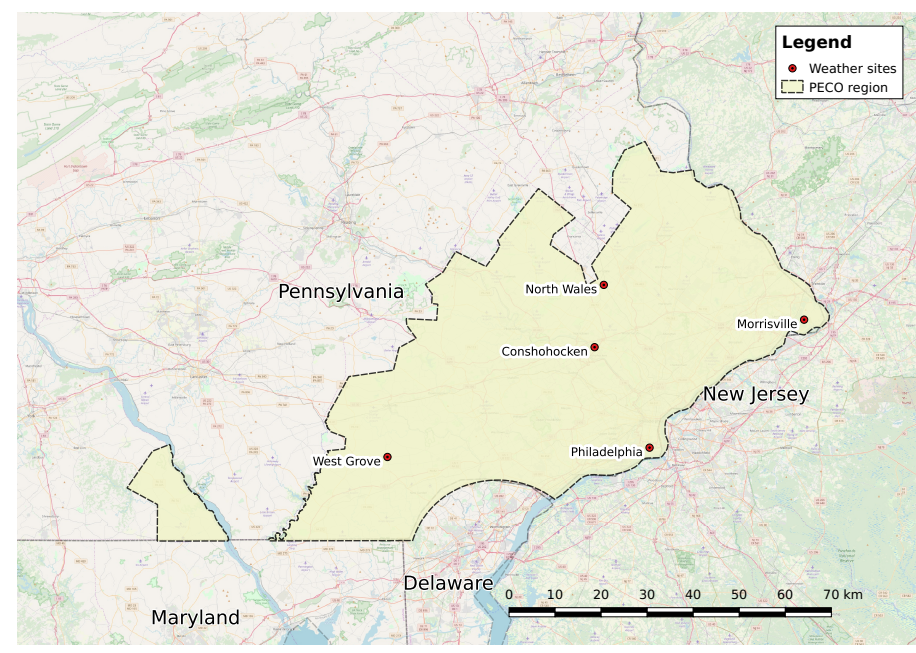

Figure 4: Map of the 5 representative weather locations. Credits: OpenStreetMap contributors, QGIS Desktop.

of renewable energy generation. The models used to estimate generation from irradiance or wind speed are detailed in the following sections. Details of the calculations for the comparison data in Oldenburg, Germany may be found in (32).

\subsubsection{Solar Power Production}

Modeling of solar PV energy production was performed using NREL PVWatts, via the open source System Advisor Model (SAM) package ${ }^{11}$. PVWatts uses a simplified model of PV generation (45) that does not account for detailed technical characteristics of the modules or inverter used. The NSRDB irradiance data were used as an input to the model. For this study, we modeled each system with a capacity of $1.2 \mathrm{MW}$ at a $20^{\circ}$ tilt, facing due south. We used the default values for the various losses computed by the tool. This resulted in a system with an annual energy yield of around $1300 \mathrm{kWh} /$ rated-kW and a capacity factor of $15 \%$. As discussed, the production at 5 sites was aggregated and normalized to its mean to represent the baseline PV generation.

\subsubsection{Wind Power Production}

The MERRA-2 wind speeds at $10 \mathrm{~m}$ were extrapolated to 100 $\mathrm{m}$ using the exponential law discussed previously. The relationship between wind speed and produced power was modeled using a simplified cubic model, as in previous work on FlexiGIS (32).

$$
W(t)= \begin{cases}P_{r} \frac{v^{3}}{v_{r}^{3}} & \text { for } v \leq v_{r} \\ P_{r} & \text { for } v_{r}<v<v_{\text {out }} \\ 0 & \text { for } v \geq v_{\text {out }}\end{cases}
$$

Here $v$ is the wind speed, $P_{r}$ and $v_{r}$ are the rated power and wind speed of the turbine respectively, and $v_{\text {out }}$ is the cutout

\footnotetext{
${ }^{11}$ https://sam.nrel.gov
}

wind speed. For this study, parameters were obtained for a Gamesa G83 2MW turbine, a model used in wind installations located in other parts of Pennsylvania ${ }^{12}$. Parameters used for this turbine were $P_{r}=2 M W, v_{r}=13.5 \frac{\mathrm{m}}{\mathrm{s}}$ and $v_{\text {out }}=25 \frac{\mathrm{m}}{\mathrm{s}}$. As with solar power, generation for the 5 sites was aggregated and normalized to its mean value. The resultant energy yield for wind was around $1400 \mathrm{kWh} / \mathrm{kW}$ rated installed capacity, with a capacity factor of around $16 \%$. The PECO region encompasses wind resources reported by the NREL Wind Prospector ${ }^{13}$ to be of Wind Power Class 1 and 2.

\section{Results and Discussion}

Referring to Eq. 5, it is clear that without renewable generation (i.e. when $\alpha$ and $\beta$ are zero), the variance reduces to the variance of the load: $\sigma_{R}^{2}=\sigma_{l}^{2}$. Though this is significant in an absolute sense, it renders comparison of renewable optimization on multiple sites difficult, because the cost function contains differences in the variability of the base load between the two sites. In other words, the variance of the load represents the cost of storage required to create a flat load time series in the absence of renewable generation. So for the purposes of results comparison, we will subtract the standard deviation of the load from the cost function. As this is simply a constant offset for each case, it has no effect on the variation of the cost with respect to renewable penetration or mix. However, it is still worth noting that the absolute value of the load variance is still of importance in representing undesirable variability in the existing load prior to renewable deployment.

The modified cost function plotted in the subsequent figures, $\sigma_{R}^{*}$, can be thought of as the degree to which renewable generation increases or decreases the need for balancing energy relative to the status quo of the existing demand.

$$
\sigma_{R}^{*}=\sigma_{R}-\sigma_{l}
$$

In establishing the context for the results, it may be useful to consider the patterns of the time series for the load, along with wind and solar generation. The mean load for Philadelphia in 2017 was observed to be $4542 \mathrm{MW}$ (annually, around $40 \mathrm{TWh}$ ). A sample hourly time series for a single week is shown in Fig. 5 b. In both cases we can see that solar and wind generation both seem roughly correlated, peaking during the day and dropping off at night (all the way to zero in the case of solar). Oldenburg exhibits more hours spent at the rated wind speed, resulting in clipping of the wind speed profile on the high end.

Referring to Fig. 6, we can also see the annual correlation between renewable generation and the load. One difference that is plainly visible is that Oldenburg exhibits a load profile that peaks during the winter months, while Philadelphia's profile retains a slight peak during winter, but also exhibits a larger peak

\footnotetext{
${ }^{12}$ https://eerscmap.usgs.gov/uswtdb/

${ }^{13} \mathrm{https}: / /$ maps.nrel.gov/wind-prospector/
} 


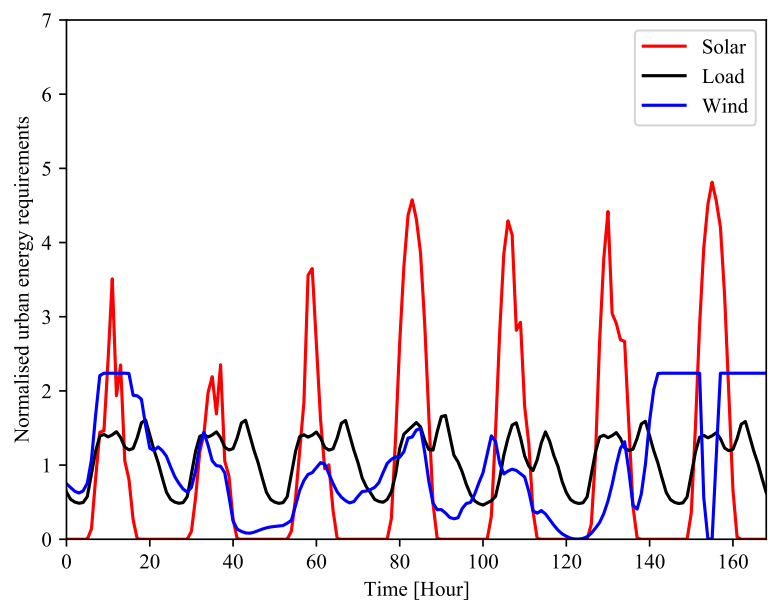

(a) Oldenburg

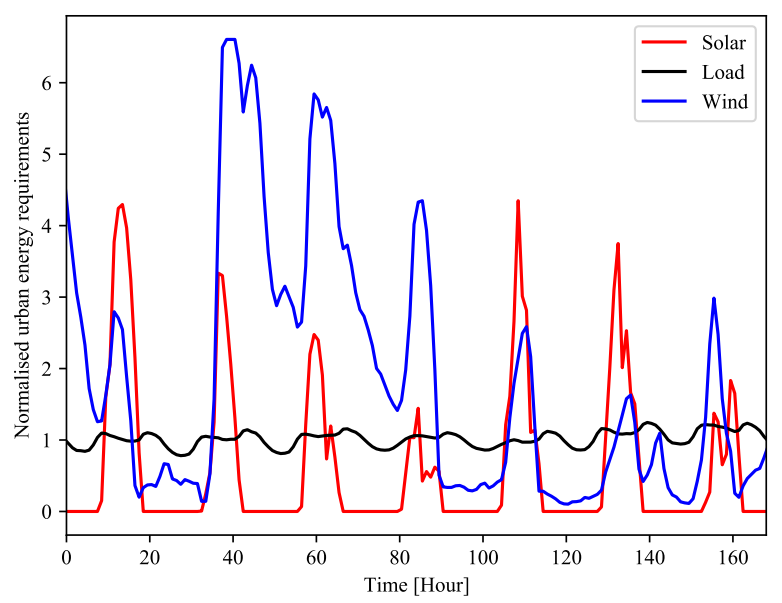

(b) Philadelphia

Figure 5: Snapshot of hourly time series of load, wind and solar generation.

during summer months, corresponding to higher air conditioning load. Both cities demonstrate wind generation that peaks during winter and declines in summer. Solar in both cases exhibits the opposite trend, peaking in the summer months, but this effect is much more pronounced in the Oldenburg case. On an annual basis, this amounts to a greater correlation between solar and load in Philadelphia, than in Oldenburg.

In Fig. 7, we plot the cost function relative to both the fraction of energy imported from the grid (i.e. $\langle C\rangle$ ), and the relative mix of solar and wind within the fraction of distributed renewable generation. Both sites show some similar characteristics. In both cases, we see that the variability is dependent on the exact mix of renewables used. For a fixed level of grid independence, Oldenburg exhibits the lowest variability when using a renewable mix of around $80 \%$ wind $20 \%$ solar, while Philadelphia favors a mix that is closer to even, around $50 \%-50 \%$. In general, we see that both sites exhibit increased variability in

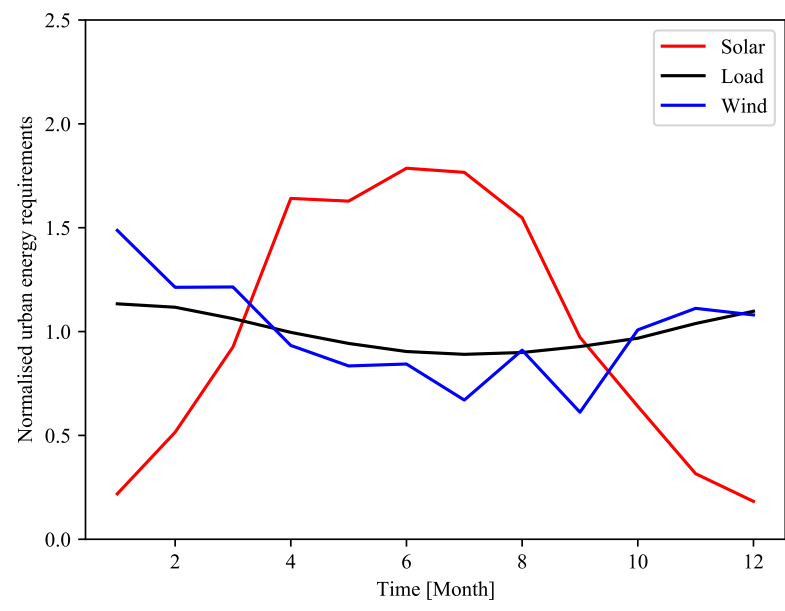

(a) Oldenburg

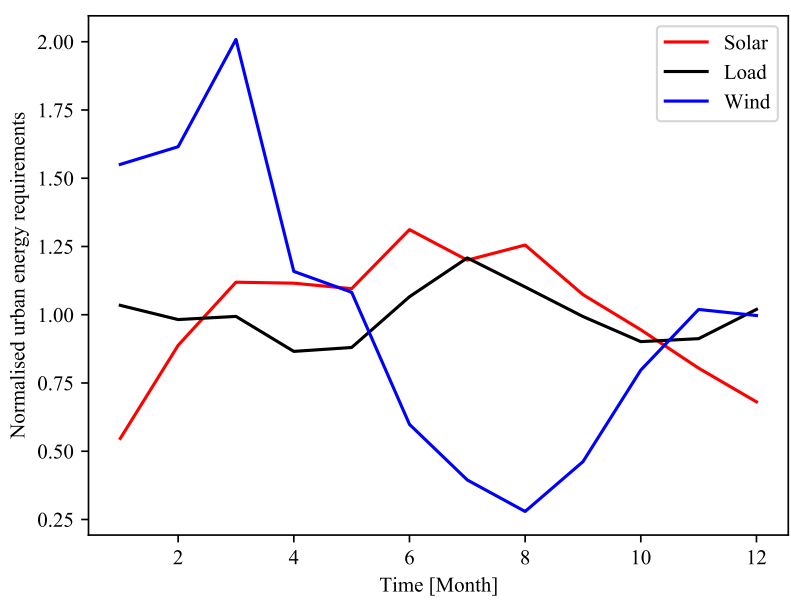

(b) Philadelphia

Figure 6: Aggregate monthly time series of load, wind and solar generation.

the residual load with decreased reliance on the grid (moving leftward on the figure). However, we can also observe that Philadelphia encounters the same levels of variability at much lower levels of grid independence, which is to say that Oldenburg can tolerate a higher renewable contribution with less need for balancing. We may partially explain this effect by considering Fig. 6. In the Oldenburg case, we see that the wind's annual profile matches well with load, while in Philadelphia, the load's combined winter and summer peaks do not match neatly with either renewable generation profile. Deeper analysis of this effect, which includes the daily variation, can be performed by considering the underlying statistical metrics from the time series.

Referring to Table 1, we can gain some mathematical understanding of the results discussed in the preceding figure. Three metrics in the table show noticeable differences between the two sites: the load in Oldenburg has a higher standard deviation than that in Philadelphia, the wind generation in Oldenburg has 


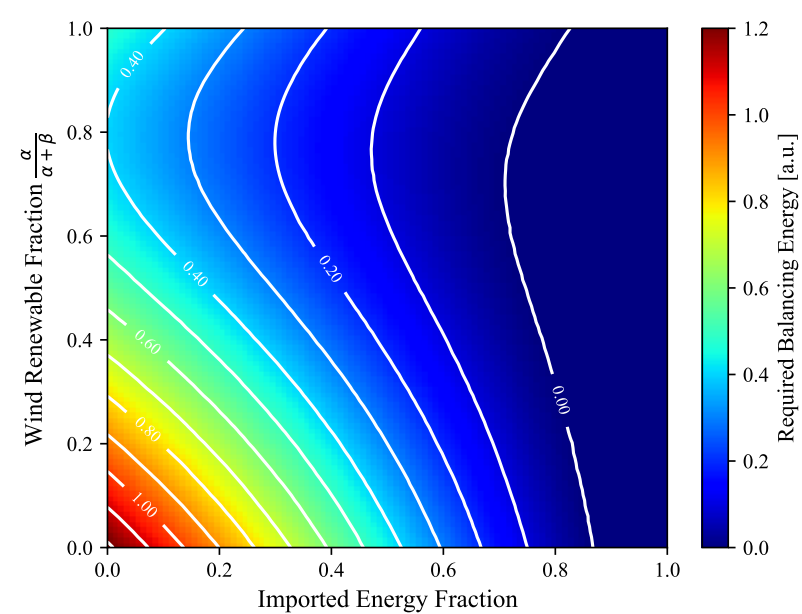

(a) Oldenburg

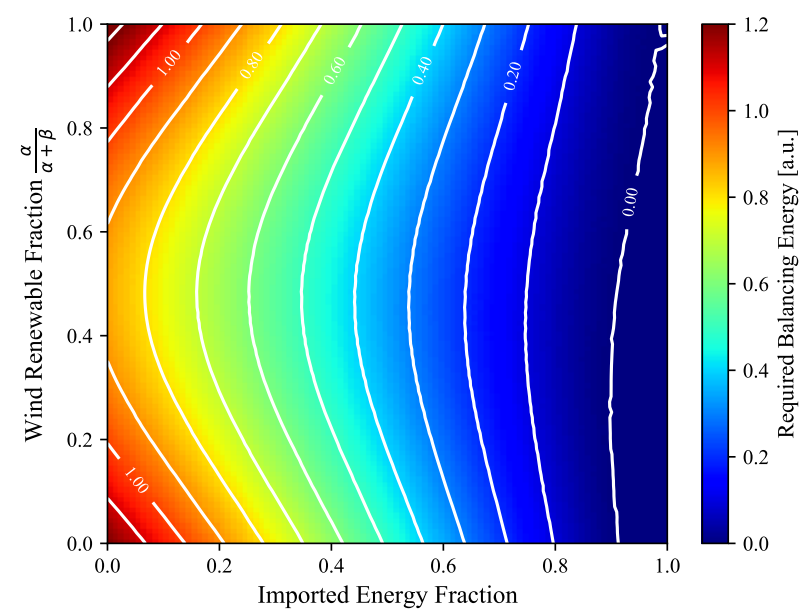

(b) Philadelphia

Figure 7: Contour plot of the required balancing energy as a function of energy imported from the grid, and the fraction of the renewable generation that is wind.

Table 1: Statistical results for the various time series, compared between the two sites. All quantities are unitless, as they are based on normalized time series.

\begin{tabular}{|l|l|l|}
\cline { 2 - 3 } \multicolumn{1}{c|}{} & Oldenburg, Germany & Philadelphia, US \\
\hline$\sigma_{l}$ & 0.340 & 0.196 \\
\hline$\sigma_{w}$ & 0.815 & 1.43 \\
\hline$\sigma_{s}$ & 1.61 & 1.44 \\
\hline$\rho_{l w}$ & 0.209 & 0.054 \\
\hline$\rho_{l s}$ & 0.141 & 0.171 \\
\hline$\rho_{s w}$ & 0.320 & 0.324 \\
\hline
\end{tabular}

a lower standard deviation than that in Philadelphia, and the wind generation in Oldenburg has a much higher correlation with the load than in Philadelphia. We can also observe that the solar energy in Oldenburg is slightly less correlated with the load than that in Philadelphia.

The analytical formulation of the optimization model allows us to confirm that these statistical metrics are consistent with characteristics that we observe in the optimal mix plot in Fig. 7. Relative to Philadelphia, Oldenburg's wind time series is both less variable and better correlated with the load, and as a result, Oldenburg's optimal mix exhibits a preference toward wind. Some basic testing of sensitivity to these metrics shows that the effect of the reduced variance is the stronger driver of this preference.

The primary interpretable result from this data is that in both the cases of Oldenburg and Philadelphia, it is possible to determine an optimal mixture of distributed renewable generation that minimizes the required balancing energy as measured by the induced variability in the residual load. Relative differences between the two cities can be identified by considering the statistical relationships between the load, wind and solar generation time series; the stronger correlation between the wind and the load time series appears to be a driver in the optimal mix for Oldenburg favoring wind generation. We also observe that Philadelphia has the same relative increase in required balancing load at around $43 \%$ imported energy (57\% renewable generation) as for the full renewable case in Oldenburg. According to the statistical model, this could primarily be attributed to increased variance in both the load and wind time series, as well as the lower correlation between wind and the load. For the models used here, meeting this scenario (approximately $28.5 \%$ of the annual load met via solar, $28.5 \%$ by wind, and $43 \%$ from other sources) would require around a rated $8.7 \mathrm{GW}$ of installed solar and a rated $8.1 \mathrm{GW}$ of wind.

From these results, we can also offer some example analysis related to energy policy. In order to minimize the requirements for additional load balancing, under current conditions, Philadelphia should favor a strategy that uses roughly an even mix of distributed solar and wind. At a level of imported energy of around $65 \%$, the balancing energy is roughly twice that required to balance out the current load ( $\sigma_{l}$ for the baseline is around 0.2 ), while it would be expected to triple the current level for a $50 \%$ renewable scenario. While balancing energy in a sustainable context could take the form of storage to provide time shifting of overgeneration, demand deficits could also be met through other forms of renewable generation that can be operated in a dispatchable manner, such as hydroelectric power or combustion of biomass.

Additional analysis may be performed through deep inspection of Eq. 5 via simple statistical terms. One strategy that may be expected to reduce the requirement for balancing load is demand management policies aimed at increasing the correlation between the load and wind or solar generation, as these correlations appear with negative coefficient in the equation. Additionally, we observe that the required balancing energy can be 
expected to decline with decreased variance in both the wind and solar time series. One example of such an approach would be reducing the rated speed of the wind turbine in the generation model, which increases the amount of time that the power output is "capped" in Eq. 7, thereby reducing the time series variance. Given that the Philadelphia region has at best a Class 2 wind resource, it may be reasonable to consider a turbine with a lower rated speed. Fig. 8 depicts a contour plot of the required balancing energy for Philadelphia subject to an alternate wind turbine performance model with a lower rated speed of $v_{r}=11 \frac{\mathrm{m}}{\mathrm{s}}$ (around $v_{\text {avg }}+6 \frac{\mathrm{m}}{\mathrm{s}}(46)$ ). We can observe that, from a meteorological point of view, the optimal ratio of wind to solar shifts to favor wind (about $60 \%$ wind, $40 \%$ solar), and we can achieve a greater degree of energy independence than in the initial case. We see a comparable balancing energy requirement at $38 \%$ imported energy for the modified turbine as with $43 \%$ imported energy in the original case.

It is important to note that this essentially amounts to curtailment that would reduce the overall power produced by a given renewable energy system. For example, because of the cubic dependence of power on wind speed, a wind turbine rated for $11 \frac{\mathrm{m}}{\mathrm{s}}$ would be expected to produce only $54 \%$ of the energy as a turbine with the same size and performance rated for $13.5 \frac{\mathrm{m}}{\mathrm{s}}$. So meeting the same fraction of the load with installed wind capacity would require a greater number of installations ( $85 \%$ more) at this lower individual turbine rating, or else would require larger (in physical size) individual installations. A similar effect (albeit shifting to favor solar) would be expected if curtailment were used to limit the variance in the solar generation. Because curtailing generation to reduce the need for storage would require extra generation, the attractiveness of these options in an absolute sense depends on the relative financial costs associated with additional storage vs. generation.

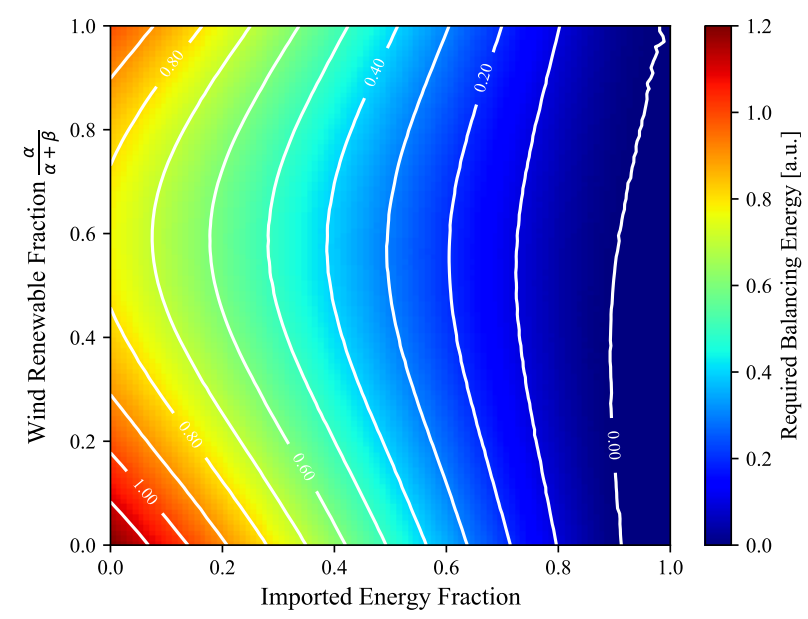

Figure 8: Required balancing energy for Philadelphia for a modeled wind turbine with reduced rated speed, $v_{r}=11 \frac{\mathrm{m}}{\mathrm{s}}$. All other conditions remain identical to previous model.

\section{Conclusions}

In this paper, we demonstrate that FlexiGIS, an open spatialtemporal analysis tool that had been developed for a European context, was able to be successfully applied to model optimization of renewable energy mix in a United States context as well. Differences in the availability of open resources between $\mathrm{Eu}-$ rope and the United States prompted different choices for the underlying data that served as the basis for the model. While OpenStreetMap was previously used as an open data source for calculations based in Europe, we found that it did not have complete coverage in the case study (about 10\% of buildings in a more complete dataset). Rather, state-based open data sources (Pennsylvania Spatial Data Access - PASDA, in this case) provided an alternative to OpenStreetMap data in the United States. Though this data was found to be more complete, the decentralized nature of the multilevel sources (e.g. state- or city-based) in the United States may require some additional work on the part of the user. Future investments by OpenStreetMap users in aggregating available data sources may unify the data availability and ease the initial time commitment in acquiring data. Regardless, in this case study, the direct availability of openly available electrical demand time series for the PECO region made the use of buildings to estimate the load superfluous.

A comparison was made between a previous European case study in Oldenburg, Germany (32), and the City of Philadelphia, Pennsylvania in the United States. Philadelphia showed a greater need for balancing energy under increasing renewable energy contribution level than did Oldenburg. Philadelphia shows similar needs at a $57 \%$ renewable contribution as Oldenburg shows under full renewable generation. On the other hand, while Oldenburg heavily favored wind generation for this optimal mix, Philadelphia had a relatively balanced optimal mixture of solar and wind to minimize the cost function. This roughly matched with expectations derived from the annual variation in load, wind and solar generation observed in the aggregate monthly time series. Recasting the cost function in terms of the individual time series standard deviations and the correlation coefficients between them allowed us to gain some additional insight from these observations, namely that the difference is primarily driven by the lower variance in the wind time series, and its greater correlation with the load. We observe that choosing a wind turbine with a lower rated speed to correspond to Philadelphia's lower wind resource class can shift the optimum towards wind, and reduce the required balancing energy, at a cost of requiring more (or larger) wind turbines to meet the equivalent fraction of the load. This result provides additional insight into the sensitivity of the model to various input parameters, and thus the "knobs" by which the results could be tuned in different scenarios.

Advancing modeling of renewable energy systems based on open data is important to enabling various energy stakeholders to make data-driven decisions in planning, policy and administration of Urban Energy Systems. This paper presents a case study by which we have broadened the scope in which FlexiGIS has been applied, and demonstrated its flexibility to work with 
alternate data sources and how it can be used to make judgments about renewable energy in a setting in the United States. Further work may consider improvements in the automation of the process and reduce the need for user-driven pre-processing of data for the analysis. Nonetheless, we believe the value in demonstrating the tool may help improve access to sophisticated analyses of Urban Energy Systems and reduce barriers to energy planning and information dissemination.

\section{Appendix}

The complete script for performing the energy mix optimization as well as sample input and output data sets are included in the supplementary material provided with the present contribution. Normalized load and renewable generation data is stored as an hourly time series in individual comma separated values (CSV) files that are read by the code. Note that the load file provided is based on a simulated residential building in Philadelphia, as PJM's data license does not allow for redistribution of the load data that was used to generate the figures in the paper.

The code (remix_calculator.py) is developed for Python 3. It requires the library numpy for calculations and uses scipy and matplotlib to generate the figures. The script is published under the 3-Clause BSD (Berkeley Standard Distribution) open source license. Required balancing energy is computed for a series of wind and solar mixtures, as well as overall renewable energy fraction. Intermediate results are saved to standard_deviations.csv. A contour plot similar to Fig. 7 is generated for an example of visualization. All developed codes and supplementary data associated with this article can be found at Github FlexiGIS_Philadelphia.

\section{Acknowledgements}

The first author acknowledges financial support by Penn State Engineering Technology and Commonwealth Engineering and Penn State Hazleton, and appreciates the Carl von Ossietzky University of Oldenburg and the DLR Institute of Networked Energy Systems for hosting his visiting scientist appointment.

\section{References}

[1] UN. 2018 Revision of World Urbanization Prospects. In: World Urbanization Prospects. United Nations; 2018. p. -. Available from: https : //population.un.org/wup/Publications/.

[2] Couture T, Busch H, Hansen T, Leidreiter A. Renewables in Cities 2019 Global Status Report - Preliminary Findings. REN21; 2019.

[3] UN. Habitat III: Third United Nations Conference on Housing and Sustainable Urban Development. Journal of Education for Sustainable Development. 2015 aug;9(2):125-125. Available from: https://doi.org/ 10.1177/F0169796x15600604c.

[4] Kraas F, Leggewie C, Lemke P, Matthies E, Messner D, Nakicenovic N, et al. Humanity on the move: Unlocking the transformative power of cities. German Advisory Council on Global Change; 2016.
[5] Sait M, Chigbu U, Hamiduddin I, de Vries W. Renewable Energy as an Underutilised Resource in Cities: Germany's 'Energiewende' and Lessons for Post-Brexit Cities in the United Kingdom. Resources. $2018 \mathrm{dec} ; 8(1): 7$. Available from: https://doi.org/10. 3390/resources 8010007 .

[6] Erhorn-Kluttig H, Jank R, Schrempf L, Dütz A, Rumpel F, Schrade J, et al. Energetische Quartiersplanung. Fraunhofer IRB, Stuttgart. 2011;

[7] DENA. Transition 19 - The biggest challenges are also the most powerful accelerators. In: Focus on urban energy transition. German Energy Agency; 2018. p. xx-xx. Available from: https://www.dena.de/fileadmin/dena/Publikationen/ PDFs/2019/transition19.pdf.

[8] of Philadelphia Office of Sustainability TC. Powering Our Future: A Clean Energy Vision for Philadelphia. The City of Philadelphia Office of Sustainability; 2018. Available from: https://www.phila.gov/documents/ powering-our-future-a-clean-energy-vision-for-philadelphia/.

[9] Stokes LC, Breetz HL. Politics in the U.S. energy transition: Case studies of solar, wind, biofuels and electric vehicles policy. Energy Policy. 2018;113:76 - 86. Available from: http://www. sciencedirect. com/science/article/pii/S0301421517307322.

[10] United Nations GA. Annual Energy Outlook 2019. US Energy Information Administration; 2019. Available from: https://www.eia.gov/ outlooks/aeo/index.php.

[11] Bagheri M, Delbari SH, Pakzadmanesh M, Kennedy CA. City-integrated renewable energy design for low-carbon and climate-resilient communities. Applied Energy. 2019 apr;239:1212-1225. Available from: https : //doi.org/10.1016/j.apenergy.2019.02.031.

[12] Radzi A. The 100\% Renewable Energy Metropolis: Governing the Design of Cities for Renewable Energy Infrastructures. In: Urban Energy Transition. Elsevier; 2018. p. 85-113. Available from: https: //doi.org/10.1016/b978-0-08-102074-6.00023-1.

[13] Çelik D, Meral ME. Current control based power management strategy for distributed power generation system. Control Engineering Practice. 2019;82:72 - 85. Available from: http://www. sciencedirect.com/ science/article/pii/S0967066118306051.

[14] Alhamwi A, Medjroubi W, Vogt T, Agert C. OpenStreetMap data in modelling the urban energy infrastructure: a first assessment and analysis. In: Proceedings of the 9th International Conference on Applied Energy. vol. 142. Elsevier; 2017. p. 1968-1976. Available from: https : //doi.org/10.1016/j.egypro.2017.12.397.

[15] United Nations GA. Transforming Our World: The 2030 Agenda for Sustainable Development. United Nations; 2015. Available from: https://www.unfpa.org/resources/ transforming-our-world-2030-agenda-sustainable-development.

[16] Ranalli J, Calvert K, Boz MB, Brownson JRS. Toward comprehensive solar energy mapping systems for urban electricity system planning and development. The Electricity Journal. 2018;31(1):8 - 15. Available from: http://www.sciencedirect.com/science/article/ pii/S1040619017303524.

[17] Alhamwi A, Medjroubi W, Vogt T, Agert C. GIS-based urban energy systems models and tools: Introducing a model for the optimisation of flexibilisation technologies in urban areas. Applied Energy. 2017 apr;191:19. Available from: https://doi.org/10.1016/j.apenergy. 2017. 01.048.

[18] Howells M, Rogner H, Strachan N, Heaps C, Huntington H, Kypreos $\mathrm{S}$, et al. OSeMOSYS: The Open Source Energy Modeling System. Energy Policy. 2011 oct;39(10):5850-5870. Available from: https: //doi.org/10.1016/j.enpol.2011.06.033.

[19] Jayadev G, Leibowicz BD, Kutanoglu E. U.S. electricity infrastructure of the future: Generation and transmission pathways through 2050. Applied Energy. 2020 feb;260:114267. Available from: https://doi.org/10. $1016 / j$. apenergy . 2019.114267.

[20] Brenna M, Dolara A, Foiadelli F, Leva S, Longo M. Urban scale photovoltaic charging stations for electric vehicles. IEEE Transactions on Sustainable Energy. 2014;5(4):1234-1241.

[21] Gastelu JV, Trujillo JDM, Padilha-Feltrin A. Hierarchical Bayesian Model for Estimating Spatial-Temporal Photovoltaic Potential in Residential Areas. IEEE Transactions on Sustainable Energy. 2017;9(2):971979.

[22] Robinson D, Haldi F, Kämpf J, Leroux P, Perez D, Rasheed A, et al. 
CitySim: Comprehensive micro-simulation of resource flows for sustainable urban planning. In: Proc. Building Simulation; 2009. p. 1614-1627.

[23] Nutkiewicz A, Yang Z, Jain RK. Data-driven Urban Energy Simulation (DUE-S): A framework for integrating engineering simulation and machine learning methods in a multi-scale urban energy modeling workflow. Applied Energy. 2018 sep;225:1176-1189. Available from: https : //doi.org/10.1016/j.apenergy .2018.05.023.

[24] Keirstead J, Samsatli N, Shah N, et al. SynCity: an integrated tool kit for urban energy systems modelling. Energy efficient cities: Assessment tools and benchmarking practices. 2010;p. 21-42.

[25] Nouvel R, BRASSEL KH, BRUSE M, DUMINIL E, COORS V, EICKER U, et al. SimStadt, a new workflow-driven urban energy simulation platform for CityGML city models. In: Proceedings of International Conference CISBAT 2015 Future Buildings and Districts Sustainability from Nano to Urban Scale. EPFL-CONF-213437. LESO-PB, EPFL; 2015. p. 889-894.

[26] Kolbe TH, Gröger G, Plümer L. CityGML: Interoperable Access to 3D City Models. In: Geo-information for Disaster Management. Springer Berlin Heidelberg; 2005. p. 883-899. Available from: https://doi . org/10.1007/3-540-27468-5_63.

[27] Robinson D, Campbell N, Gaiser W, Kabel K, Le-Mouel A, Morel N, et al. SUNtool - A new modelling paradigm for simulating and optimising urban sustainability. Solar Energy. 2007 sep;81(9):1196-1211. Available from: https://doi.org/10.1016/j.solener.2007.06.002.

[28] BOLLINGER LA, Evins R. HUES: A Holistic Urban Energy Simulation platform for effective model integration. In: Proceedings of International Conference CISBAT 2015 Future Buildings and Districts Sustainability from Nano to Urban Scale. EPFL-CONF-213425. LESO-PB, EPFL; 2015. p. 841-846.

[29] Dorfner J. rivus: A mixed integer linear optimisation model for energy infrastructure networks. Technical University of Munich. 2018;Available from: https://media.readthedocs.org/pdf/urbs/latest/ urbs.pdf.

[30] Fuchs M, Teichmann J, Lauster M, Remmen P, Streblow R, Müller D. Workflow automation for combined modeling of buildings and district energy systems. Energy. $2016 \mathrm{dec} ; 117: 478-484$. Available from: https : //doi.org/10.1016/j.energy.2016.04.023.

[31] Alhamwi A, Medjroubi W, Vogt T, Agert C. Development of a GIS-based platform for the allocation and optimisation of distributed storage in urban energy systems. Applied Energy. 2019 oct;251:113360. Available from: https://doi.org/10.1016/j.apenergy.2019.113360.

[32] Alhamwi A, Medjroubi W, Vogt T, Agert C. Modelling urban energy requirements using open source data and models. Applied Energy. 2018 dec;231C:1100-1108. Available from: https://doi.org/10.1016/ j.apenergy . 2018.09.164.

[33] Alhamwi A, Medjroubi W, Vogt T, Agert C. FlexiGIS: an open source GIS-based platform for the optimization of flexibility options in urban energy systems. Energy Procedia. 2018;152C:944-949. Available from: https://doi.org/10.1016/j.egypro.2018.09.097.

[34] U S Census Bureau PD. Annual Estimates of the Resident Population: April 1, 2010 to July 1, 2018. U.S. Census Bureau; 2019.

[35] Bureau USC. TIGER/Line Shapefiles: Special Release - Census Blocks with Population and Housing Counts. U.S. Census Bureau; 2010. Available from: https://www.census.gov/geographies/ mapping-files/time-series/geo/tiger-line-file.2010.

html.

[36] PJM. Data Miner 2 - Hourly Load: Metered. PJM; 2019. Available from: http://dataminer2.pjm.com/feed/hrl_load_ metered/definition.

[37] Sengupta M, Xie Y, Lopez A, Habte A, Maclaurin G, Shelby J. The National Solar Radiation Data Base (NSRDB). Renewable and Sustainable Energy Reviews. 2018;89:51 - 60. Available from: http://www.sciencedirect.com/science/article/pii/ S136403211830087X.

[38] Draxl C, Clifton A, Hodge BM, McCaa J. The Wind Integration National Dataset (WIND) Toolkit. Applied Energy. 2015 08;151:355-366.

[39] Gelaro Rea. The Modern-Era Retrospective Analysis for Research and Applications, Version 2 (MERRA-2). Journal of Climate. 2017;30(14):5419-5454.

[40] Alhamwi A, Kleinhans D, Weitemeyer S, Vogt T. Moroccan National Energy Strategy reviewed from a meteorological perspective. Energy Strat- egy Reviews. 2015 jan;6:39-47. Available from: https://doi.org/ 10.1016/j.esr.2015.02.002.

[41] Bremen Lv, Knorr K, Lange B, Bofinger S. A full renewable power supply scenario for Europe: The weather determines storage and transport. In: 8th International Workshop on Large Scale Integration of Wind Power into Power Systems as well as on Transmission Networks for Offshore Wind Farms, Bremen. energynautics; 2009. p. 0-0.

[42] Heide D, von Bremen L, Greiner M, Hoffmann C, Speckmann M, Bofinger S. Seasonal optimal mix of wind and solar power in a future, highly renewable Europe. Renewable Energy. 2010 nov;35(11):2483-2489. Available from: https://doi.org/10.1016/j.renene.2010.03.012.

[43] Heide D, Greiner M, von Bremen L, Hoffmann C. Reduced storage and balancing needs in a fully renewable European power system with excess wind and solar power generation. Renewable Energy. 2011 sep;36(9):2515-2523. Available from: https://doi.org/10.1016/ j.renene. 2011.02.009.

[44] Bañuelos-Ruedas F, Ángeles-Camacho C, Rios-Marcuello S. xx. In: Methodologies Used in the Extrapolation of Wind Speed Data at Different Heights and Its Impact in the Wind Energy Resource Assessment in a Region. IntechOpen; 2011. p. xx.

[45] Dobos AP. PVWatts Version 5 Manual. National Renewable Energy Lab; 2014. Available from: https://www.nrel.gov/docs/fy14osti/ $62641 . p d f$.

[46] Bak C. 3. In: Aerodynamic design of wind turbine rotors. Elsevier Science \& Technology; 2013. p. $59-108$. 